\title{
FUNGOS ISOLADOS DO AR E DO PISO DE AMBIENTES FECHADOS DO HOSPITAL ESCOLA DA UNIVERSIDADE FEDERAL DE PERNAMBUCO, RECIFE, BRASIL - I
}

\author{
María da Glória de Barros \\ Departamento de Farmácia do Centro de \\ Ciências de Saúde da Universidade Federal \\ de Pernambuco Av. Artur de Sá S/N, \\ Cidade Universitária 50.740, \\ Recife-Pernambuco, Brasil.
}

\author{
M. A. Q. Cavalcanti, D. M. Massa Lima \\ e M. J. S. Fernandes \\ Departamento de Micología do Centro \\ de Ciências Biológicas da Universidade \\ Federal de Pernambuco Av. Artur de Sá, S/N, \\ Cidade Universitária 50.740, \\ Recife-Pernambuco, Brasil
}

Palabras clave: Hospital, Hongos ambientales, saprófitos y oportunistas.

Key words: Hospital, environment, saprophytic and opportunistic fungi.

\section{RESUMO}

Do ar e do piso de ambientes fechados do Hospital das Clínicas da Universidade Federal de Pernambuco, Recife, Brasil, foram isoladas 3.330 colônias de fungos que corresponden a 123 entidades taxonômicas pertencentes em sua maioria aos Asco-Deuteromycotina (95,3\%), estando os demais grupos pouco representados Zigomycotina $(5,6 \%)$ Basidiomycotina $(0,8 \%)$ e Micelia sterilia $(0,2 \%)$. Nâo houve diferença significativa entre o quantitativo de colônias detectadas durante o periodo de pluviosidade e estiagem. Observou-se maior número de colônias no piso em confronto com as detectadas no ar atmosférico. Os gêneros encontrados com mais freqüência e maior número de espécies foram Aspergillus, Penicillium $e$ Fusarium. Dentre os Hyphomycetes isolados 14 espécies sâo referidas como agentes etiológicos de micoses.

\section{RESUMEN}

[Hongos aislados del aire y del piso en ambientes confinados en el Hospital Escola Da Universidade Federal de Pemambuco, Recife, Brasii. I]

Del aire y del piso de ambientes confinados del "Hospital das Clinicas da Universidade Federal de Pemambuco, Recife, Brasil", fueron aisladas 3.330 colonias de hongos correspondientes a 123 entidades taxonómicas pertenecientes en su mayoría a los AscoDeuteromycotina $(95,3 \%)$, estando los demás gnt- pos escasamente representados Zigomycotina $(5,6 \%)$; Basidiomycotina $(0,8 \%)$ y Micelia sterilia $(0,2 \%)$. No hubo diferencias significativas entre la cantidad de colonias aisladas durante los periodos de pluviosidad y verano. Observándose el mayor número de colonias en el piso más que en aquellas aisladas del aire atmosférico. Los géneros encontrados con mayor frecuencia fueron Aspergillus, Penicillium y Fusarium. Entre los Hyphomycetes aislados, 14 especies son referidas como agentes etiológicos de micosis.

\section{SUMMARY}

[Fungi isolated from air and floor in closed environment in the Hospital Escola Da Universidade Federal de Per. nambuco, Recife, Brasil. I.]

The air and floor in hospital environment of the Clinical Hospital of the Federal university of Pernambuco, Recife, Brasil, were isolated 3.330 colonies corresponding to 123 taxonomic entities, most of them of Deuteromycotina group $(95,3 \%)$, being the other groups little represented Zygomycotina (5,6\%), Basidiomycotina $(0.8 \%)$ and Mycelia sterilia $(0.2 \%)$. There was no meaningful difference between the colonies isolated during the rainy and dry period. Grater number of colonies was observed from the material collected on the floor, rather than from the air. The most frequent genera and most number of species were: Aspergillus, Penicillium and Fusarium. Through Hyphomycetes isolated 14 species are refered as mycoses agents. 


\section{INTRODUÇĀO}

As pesquisas visando o reconhecimento de fungos presentes na atmosfera, tê-m interessado a especialistas de diferentes países como Negroni \& Fischer (1942), Argentina: Oliveira Lima (1941), Machado (1952), Alecrin \& Teixeira (1958), Gambale et al. (1977), Lima \& Gadelha (1983), Brasil; Grose et al. (1967), Colômbia; Evans \& Ruiz (1955), Costa Rica; Taylor \& McFadden (1962), Panamá; Montemayor \& Gamero (1962), Romero \& Rincón (1969), Venezuela.Estes trabalhos estâo relacionados com torre de igreja, praças, pôlos industriais e margens de rios.

Silva et al. (1983) isolaram fungos de vârios ambientes de um Hospital Escola de Belo Horizonte, MG, Brasil; os ambientes variaram quanto â finalidade e circulaçâo de pessoas; neste trabalho, assim como nos outros acima mencionados, os taxa isolados foram identificados apenas a nivel de gênero.

A patologia por fungos oportunistas adquire na prâtica mêdica importância cada vez maior em ambientes hospitalares e serviços especializados, sendo considerados inclusive os aspectos referentes a: prôteses cardíacas e transplantes de rins; diabete; tuberculose, neoplasias e outras enfermidades graves; uso indiscriminado de antibióticos, esteróides e imunodepressores e outros aspectos considerados como fatores predisponentes de infecçôes micóticas, Davis (1974), Teixeira (1979), Rippon (1982), Moraes et al. (1984), Lacaz (1984).

$\mathrm{O}$ exposto justifica o presente trabalho, que foi desenvolvido em ambientes fechados (sem acesso ao público) do Hospial das Clínicas da Universidade Federal de Pernambuco (UFPE), Recife, PE, Brasil, e teve como objetivos: isolar e identificar a nivel de espécie, fungos do ar e do piso desses ambientes; relacionar os dados obtenidos com os períodos de pluviosidade e estiagem; comparar os resultados com os obtidos por outros autores.

Neste trabalho, apresenta-se apenas os resultados obtenidos referentes aos fungos filamentosos.

\section{MATERIAL E METODOS}

O Hospital das Clínicas da UFPE, Recife, Brasil, como Hospital Escola, além de ser instituiçâo de ensino, possui grande diversidade de ambientes e intensa movimentaçâo humana. Entre os ambientes fechados ao público, foram selecionados: Berçârio (B), Sala de Parto (SP), Bloco
Cirúrgico (BC), Diálise Peritonial e Hemodiâlise (DPH), Unidade de Terapia Intensiva (UTI), Sala de Material Cirúrgico Esterilizado (SMCE), Bloco de Pequeña Cirurgia (BPC), Laboratôrio de Análises Clínicas (LAC).

Foram considerados os períodos climáticos de plúviosidade (julho e agosto/85) e estiagem (dezembro/85 e janeiro/86). Em intervalos quinzenais nos meses de julho, agosto e dezembro de 1985 e janeiro de 1986, procederam-se a um total de 8 coletas, sempre no expediente da manhâ, após a limpeza habitual dos ambientes predeterminados.

Para isolamento de fungos foi utilizado o meio ágar Sabouraud adicionado de $50 \mathrm{mg} / 1$ de Cloranfenicol e contido em placas de Petri de $5 \mathrm{~cm}$ de diâmetro. Para coleta dos propâgulos de fungos presentes no ar atmosférico dos ambientes, as placas contendo o meio foram expostas por 15 minutos, a uma altura de $1 \mathrm{~m}$ do piso; o material do piso foi coletado com cotonetes esterilizados, em movimento de zigue-zague a imediatamente semeado na superfície do meio em placas; nos dois tipos de coletas, o número de placas variou de 2 a 4 , conforme a dimensâo dos ambientes. Após as coletas, as placas foram vedadas com fita adesiva, transportadas ao Departamento de Micologia, $\mathrm{CCB}$, UFPE e deixadas à temperatura ambiente $\left(26^{\circ} \mathrm{C} \pm 2^{\circ} \mathrm{C}\right)$. Após o desenvolvimento, as colônias foram repicadas para meios específicos (contidos em tubos), de acordo com as exigências fisiológicas de cada grupo de fungos, os quais foram identificados utilizando-se entre outros, os autores Raper \& Thom (1949), Lodder (1970), Booth (1971), Ellis (1971). Raper \& Fennel (1977), Samson (1979), Stolk \& Samson (1983), Nelson (1983), Kreger-van Rij (1984), Pitt (1985), Barnet et al (1986).

\section{RESULTADOS E DISCUSSÃO}

Através de 464 exposiçôes de placas de Petri em 8 diferentes ambientes fechados do Hospital das Clínicas da Universidade Federal de Pernambuco, foram isoladas 3.330 colônias de fungos, que correspoderam a 123 entidades taxonômicas. Em relaçâo ao número desses isolamentos, a maioria pertencente subdivisâo Deuteromycotina $(90,6 \%)$ com 4 gêneros, Zigomycotina $(3,6 \%) 2$ gêneros, representada por 35 gêneros, seguindo-se Ascomycotina (47\%) com 4 gêneros Basidiomycotina $(0,8 \%)$ e $(0,2 \%)$ de Micelia sterilia.

Quanto ao número de colônias assinaladas no ar 1.195 e no piso 2.135 , verificou-se maior quantitativo no piso. Esse achado nâo coincide com o 
citado por Silva et al. (1983), sendo provável que a técnica de coleta utilizada para captar propágulos do piso, assim como, outros fatores (fluxo humano, açâo de desinfetantes etc.) tenham contribuído para diferentes resultados.

Tomamdo-se por base o número de isolamentos os gêneros foram agrupados em categorias de freqüência como o usado por Yadav \& Madelin (1968). Por essa classificaçâo os gêneros foram considerados: muito comuns $(80-100 \%)$; comuns $(61-80 \%)$; freqüentes (41-60\%); ocasionais (21$40 \%$ ) e raros (01-20\%), Tabela 1.

Dentre os Deuteromycotina, classe Hyphomycetes, foram detectadas 14 espécies que sâo referidas como agentes de micoses. Essas espécies patogénicas e/ou oportunistas abrangeram um total de 1.137 colónias isoladas, distribuídas nos 8 ambientes de coletas (Tabela 2). Observou-se que nessas espêcies nâo houve diferença significativa entre o quantitativo de colônias detectadas durante os períodos de pluviosidade e estiagem. No entanto, observou-se resultado bastante expressivo quanto ao número de colônias assinaladas entre as espécies. Neste contexto, Pathak \& Pady (1965), afirman que os esporos fúngicos estâo bem adaptados á disseminaçâo no ar atmosférico. Por outro lado, ocorrem no ar mudanças de populâo fúngica de estaçâo para estaçâo e de dia para dia, Pady (1957) e eté mesmo de hora para hora (Hirst, 1953; Panser et al. 1957 e Pady et al. 1962).

Do total de isolamentos (3.330 colônias), constatado em percentual de $51,7 \%$ para os géneros Aspergillus e Penicillium (1722 colônias), apresentando-se os demais taxa ( 39 gêneros) com $48,3 \%$ de colônias detectadas (1.608 colônias), Tabela 3.

Aspergillus e Penicillium foram tambén os taxa que se apresentaram com maior assinalamento de espécies (29 e 30 espécies respectivamente). Observou-se uma diferença significante no número de assinalamento de colônias em relaçâo á algumas espécies isoladas. Por exemplo: Aspergillus caespitosus e A. flavus representaram-se por 189 e 171 colônias respectivamente, enquanto que, $\mathbf{A}$. flavusfurcatis e $\mathbf{A}$. candidus estiveram presentes apenas com 1 colônia. Verificou-se também o assinalamento de Penicillium frequentans com 78 colônias e P. fellutanum com 1 colônia (Tabela 4). Algumas espécies tanto de Aspergillus como de Penicillium, só ocorreram no período de pluviosidade como: Aspergiflus alliaceus, A. flavo-furcatis, A. terricola, Penicillium capsulatum, P. rasitrickii, P. velutinum, enquanto que: Aspergillus avenaceus, A. awamorii, A. candidus, A repens, Penicillium capsulatum, P. decumbens, $P$. funiculosum e $P$. simplicissimum foram assinalados somente no período de estiagem.
Aspergillus é referido por Negroni \& Negroni (1984), como sendo componente habitual da micota anemófila. Dransfield (1966) afirma ser esse gênero mais freqüente em regiôes quentes e úmidas, nâo apresentando clara periodicidade estacional. A. flavus, A. fumigatus, A. niger, A. sydowii e A. terreus, encontrados neste estudo com grande freqüência, sâo também referidas em pesquisas semelhantes por Kramer et al. (1960), Moustafá \& Kamel (1976), Calvo et al. (1980 a), Abdel-Hafez \& Shoreit (1985) e Abdez- Hafez et al. (1986). Por outro lado, A. flavus, A niger, A. ochraceus, A. oryzae, A.parasiticus e A. ruber, sâo mencionadas por Austwick (1965), Diener \& Davis (1969), Tango (1974), Chu (1974), Torras er al. (1979) e Dorner et al. (1984), como produtoras de toxinas. A. tamarii foi também isolada do ar atmosférico por Abdel-Hafez \& Shoreit (1985), citando os referidos autores ser a mesma produtora de ácido kójico.

Outro aspecto que deve ser considerado, é que certas espécies de Aspergillus têm importância na micopatologia humana. Algumas, atacam o sistema respiratôrio causando alergia e rinites (Sandhu \& Sandhu, 1973; Raper \& Fennel, 1977; Lacaz et al., 1984 ), outras causam aspergiloma pulmonar (Gemeinhardt, 1969; Safelder, 1980; Landerker, 1982), enquanto outras atingem o sistema circulatório (Haddorn, 1960; Drexler et al., 1980). Negroni et al. (1972) citam que algumas espécies de Aspergillus produzem otomicoses. A. fumigatus, A. niger e A. navus, encontradas com freqüência nesta pesquisa, sâo referidas por Sepúlveda (1985), como espécies mais isoladas, em casos de infecçôes humanas.

Penicillium destaca-se juntamente com Aspergillus em pesquisas de fungos do ar atmosférico. P. fellutanum $P$. frequentans, $P$. purpurogenum e $P$. steckii, isoladas comumente nesta pesquisa, sâo também citadas entre outras espécies em pesquisas de fungos anemófilos por Kramer et. al., 1960; Moustafá \& Kamel, 1976; Barkai-Golan et al., 1977 e Abdel-Hafez et al., 1986. Quanto á capacidade de produçâo de toxinas, algunas espécies encontradas nesta investigaçâo como P. frequentans, P. citrinum e P. viridicatum, sâo reportadas por Tango (1974) e Chu (1974), como productoras de ochratoxinas. P. rubrum e P. islandicum, citadas respectivamente por Newberne, 1974; Esteves et al., 1977, como productoras de rubratoxina e islandotoxina, foram também isoladas nesta pesquisa. No campo da micopatologia humana, P. frequentans encontrada com freqüência ( 78 colônias) é referida por Cortez \& Avila (1973, 1974) e Avila \& Lacey (1974), como agente de alveolite e processos asmáticos. P. simplicissimum e $\mathbf{P}$. rubrum, encontradas também no nosso estudo, sâo citadas por Rippon (1982), como espécies predominantemente alegênicas. 
Fusarium foi outro género que se destacou em número de espécies (6) e colônias detectadas (227). Quanto as dados climáticos, F. lateritium e F. moniliforme foram comuns aos dois períodos estacionais; F. equiseti só ocorreu no período de estiagem, enquanto $\mathbf{F}$. oxysporum, F. poae e F. solani, tiveram $100 \%$ de freqüência somente no período de pluviosidade (Tabela 5).

Em pesquisas aeroesporológicas realizadas no Brasil, esse género é citado por vários autores, entre eles Lima \& Gadelha (1983), Silva et al. (1987) e Purchio et al. (1984).

Caretta et al. (1983), na Itâlia e Abdel-Hafez \& Shoreit (1985), na Arâbia, isolaram do ar atmosfé- rico, F. moniliforme, F. oxysporum e F. solani que foram tambêm detectadas nesta pesquisa. Vale ressaltar que algumas espécies de Fusarium encontradas nesta investigaçâo, sâo productoras de toxinas como, F. moniliforme - moniliformim - (Marasas et al. 1986), F. solani -zearalenone- Vesonder et al. 1981), F. equiseti - toxina $T_{2}$ - (Richardson et al. 1985).Infecçöes micóticas causadas por espécies de Fusarium podem ocorrer em pacientes com queimaduras extensas (Abramowsky et al. 1974); em casos de leucemias (Chu et al., 1974); em queratites (Kidd \& Wolf, 1973 e Laverde et al., 1973); em úlcera da pele (Zapater et al. 1972 e English, 1972); em casos de onicomicoses (Zaias, 1966) e de granuloma facial (Benjamin et al., 1970).

\section{Tabela 1}

Categoria de freqüência dos gêneros de fungos no ar e piso isolados em ambientes fechados do Hospital das Clínicas da Universidad Federal de Pernambuco.

\begin{tabular}{|c|c|c|}
\hline Muito comuns (81-100\%) & $\begin{array}{l}\text { Aspergillus } \\
\text { Cladosporium } \\
\text { Curvularia } \\
\text { Fusarium } \\
\text { Geotrichum }\end{array}$ & $\begin{array}{l}\text { Monilia } \\
\text { Paecilomyces } \\
\text { Penicillium } \\
\text { Periconia } \\
\text { Rhizopus }\end{array}$ \\
\hline Comuns $(61-80 \%)$ & $\begin{array}{l}\text { Brettanomyces } \\
\text { Candida } \\
\text { Chaetomium }\end{array}$ & $\begin{array}{l}\text { Nigrospora } \\
\text { Phoma } \\
\text { Humicola }\end{array}$ \\
\hline Freqüentes (41-60\%) & $\begin{array}{l}\text { Pestalotia } \\
\text { Xylaria }\end{array}$ & $\begin{array}{l}\text { Pithomyces } \\
\text { Torula }\end{array}$ \\
\hline Ocasionais $(21-40 \%)$ & $\begin{array}{l}\text { Acremonium } \\
\text { Alternaria } \\
\text { Aureobasidium } \\
\text { Malbranchea } \\
\text { Microascus }\end{array}$ & $\begin{array}{l}\text { Rhodotorula } \\
\text { Tilachlidium } \\
\text { Trichoderma } \\
\text { Trichosporon }\end{array}$ \\
\hline Raros $(01-20 \%)$ & $\begin{array}{l}\text { Chrysosporium } \\
\text { Cladosporiella } \\
\text { Cylindrocarpon } \\
\text { Drechslera } \\
\text { Monodictys } \\
\text { Mucor }\end{array}$ & $\begin{array}{l}\text { Arthrinium } \\
\text { Ramichloridium } \\
\text { Scolecobasidium } \\
\text { Sporothrix } \\
\text { Thielavia } \\
\text { Tritirachium }\end{array}$ \\
\hline
\end{tabular}


Fungos isolados do ar e do piso de ambientes fechados M.G. de Barros y col.

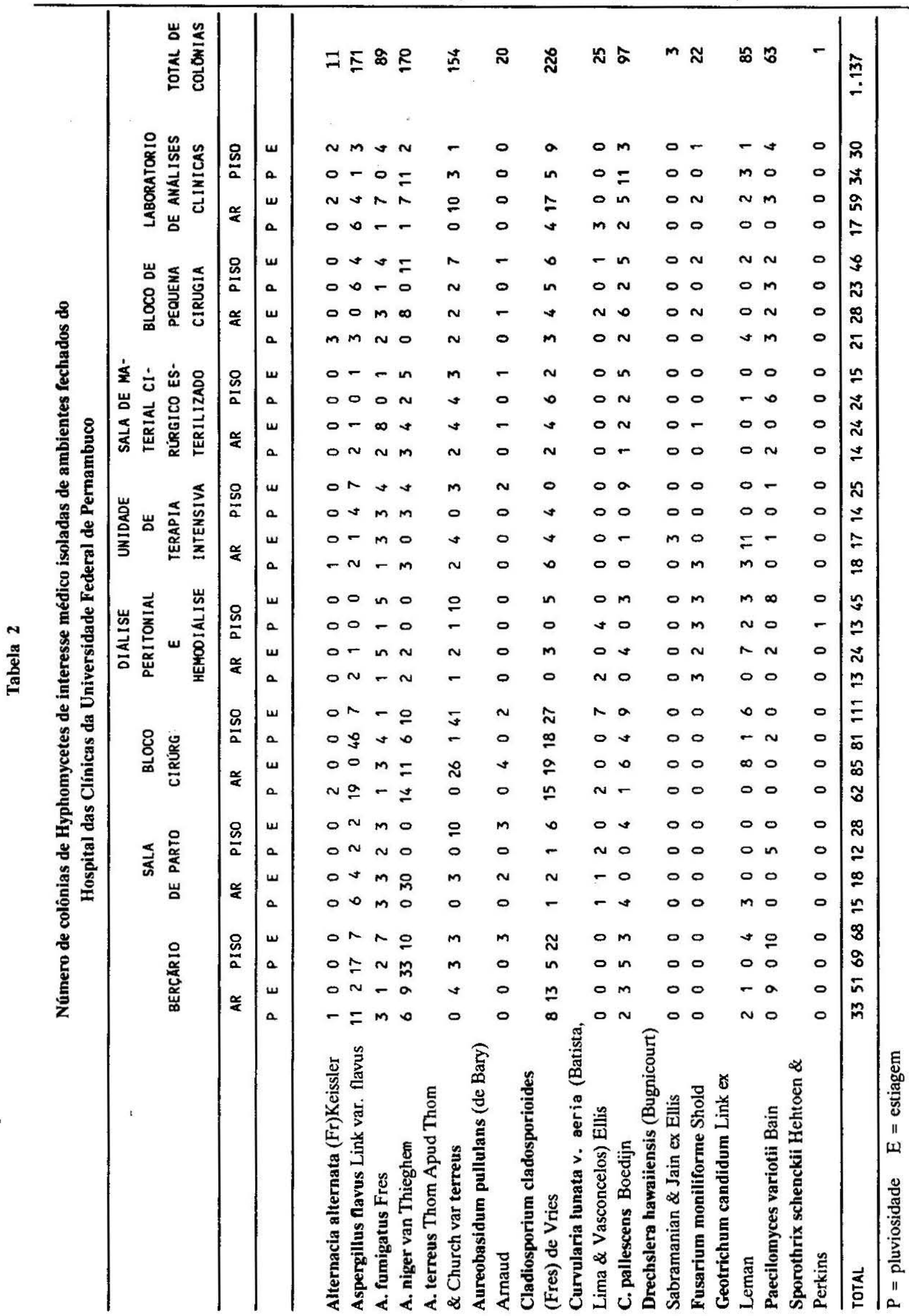


Tabela 3

Fungos isolados do ar e do piso de ambientes fechados do Hospital das Clínicas da Universidade Federal de Pernambuco.

\begin{tabular}{|c|c|c|}
\hline GENEROS & $\begin{array}{r}N^{2} \mathrm{DE} \\
\text { ESPECIES }\end{array}$ & $\begin{array}{r}\text { No DE } \\
\text { ISOLAMENTOS }\end{array}$ \\
\hline Acremonium Link & 2 & 6 \\
\hline Alternaria Ness ex Fr. & 2 & 16 \\
\hline Aspergillus Mich ex Fr. & 29 & 1.187 \\
\hline Aureobasidium Viala \& Boyer & 1 & 20 \\
\hline Brettannomyces Kufferath \& van Laer & 2 & 16 \\
\hline Candida Berkhout & 8 & 31 \\
\hline Chaetomium Kunze ex Fr. & 1 & 40 \\
\hline Chrysosporium Corda & 1 & 1 \\
\hline Cladosporiella Deighton & 1 & 3 \\
\hline Cladosporium Link ex Fr. & 2 & 274 \\
\hline Cylindrocarpon Wollenw. & 1 & 1 \\
\hline Curvularia Boedijn & 3 & 126 \\
\hline Drechslera Ito & 1 & 3 \\
\hline Fusarium Link ex Fr. & 6 & 227 \\
\hline Geotrichum Link ex Leman & 1 & 85 \\
\hline Humicola Traaen & 1 & 37 \\
\hline Malbranchea Sacc. & 3 & 3 \\
\hline Microascus Zukal & 1 & 32 \\
\hline Chrysonilia v. Arx & 1 & 83 \\
\hline Monodicyts Hughes & 1 & 12 \\
\hline Mucor Mich ex St. Am. & 1 & 54 \\
\hline Nigrospora Zimmerm & 1 & 19 \\
\hline Paecilomyces Bain & 3 & 82 \\
\hline Penicillium Link ex Fr & 30 & 535 \\
\hline Periconia Tode ex Fr. & 1 & 55 \\
\hline Pestalotia de Not (= Pestalotiopsis) & 1 & 8 \\
\hline Phoma Scc & 3 & 58 \\
\hline Pithomyces Berk \& Br. & 1 & 36 \\
\hline Arthrinium Kunze ex $\mathrm{Fr}$ (= Pteroconium) & 1 & 1 \\
\hline Ramichloridium Stahel ex de Hoog & 1 & 2 \\
\hline Rhizopus Ehrenb. & 1 & 101 \\
\hline Rhodotorula Harrison & 2 & 10 \\
\hline Scolecobasidium Abbott & 1 & 1 \\
\hline Sporothrix Hektoen \& Perkins & 1 & 1 \\
\hline Thielavia Zopf & 1 & 8 \\
\hline Tilachlidium Preuss & 1 & 6 \\
\hline Torula Pers ex Fr. & 1 & 35 \\
\hline Trichoderma Pers ex Fr. & 1 & 44 \\
\hline Trichosporon Behrend & 1 & 1 \\
\hline Tritirachium Limber' & 1 & 2 \\
\hline Xylaria Hill ex Grev & 1 & 26 \\
\hline Basidiomycotina (Grupo) & . & 26 \\
\hline Micelia sterilia (Grupo) & - & 16 \\
\hline TOTAL & & 3.330 \\
\hline
\end{tabular}


Tabela 4

Espécies de Aspergillus e Penicillium isoladas do ar e do piso de ambientes fechados do Hospital das Clínicas da Universidade Federal de Pernambuco.

\begin{tabular}{lrrr}
\hline & $\mathrm{N}^{2} \mathrm{DE}$ & $\mathrm{N}^{2} \mathrm{DE}$ \\
ESPECIES & COLONIAS & ESPECIES & COLONIAS \\
\hline
\end{tabular}

Aspergillus japonicus Saito var. aculeatus(Iizuka) Al-Musallam

A. alliaceus Thom \& Church

A. āsperescens Stolk

A. avenaceus Smith

A. awamori Nakazawa

A caesiellus Saito

A. caespitosus Raper \& Thom

A. candidus Link

A. flavo-furcatis Batista \& Maia

A. flavus Link var flavus

A. fumigatus Fresenius

A. gracilis Bainier

A. janus Raper \& Thom

A. japonicus Saito

A. niger van Tieghem

A. niveus Blochwitz

A. ochraceus Wilhelm

A. oryzae (Ahlburg) Cohn var. oryzae

A.parasiticus Speare

A. repens (de Bary) Fischer

A. ruber (Koning, Spick \& Brem) Thom

\& Church

A. sydowii (Bain \& Sant.) Thom

\& Church

A. sclerotiorum (Huber)

A. sulphureus (Fres.) Thom \& Church

A. tamarii Kita

A. terreus Thom Apud

Thom \& Church var. terreus

A. terricola Marchal var. terricola

A. ustus (Bain) Thom \& Church

A. versicolor (Vuill) Tiraboschi

Penicillium dodgei Pitt

(= P. brefeldianum)

P. brevi-compactum Dierckx

P. capsulatum Raper \& Fennell

P. chrysogenum Thom

P. citreonigrum Dierckx

(= P. citreo - viride )
P. citrinum Thom

P. corylophylum Dierckx

P. decumbens Thom

3

1

12

1

1

13

189

1

1

171

89

1

23

52

170

13

16

11

33

3

3

P. fellutanum Biourge

P. funiculosum Thom

P. glabrum (Wehmer) Westling

( = P. frequentans)

P. hirsutum Dierckx

P. implicatum Biourge

P. islandicum Sopp

P. janczewskii Zaleski ( = P. nigricans)

P. levitum Raper \& Fennell

P. lividum Westling

P. oxalicum Carrie \& Thom

P. purpurogenum Stoll

P. raistrickii Smith

P. restrictum Gilman \& Abbott

P. simplicissimun (Oudem) Thom

P. spinulosum Thom

P. thomi Maire

P. velutinum van Beyma

P. viridicatum Westling

78 


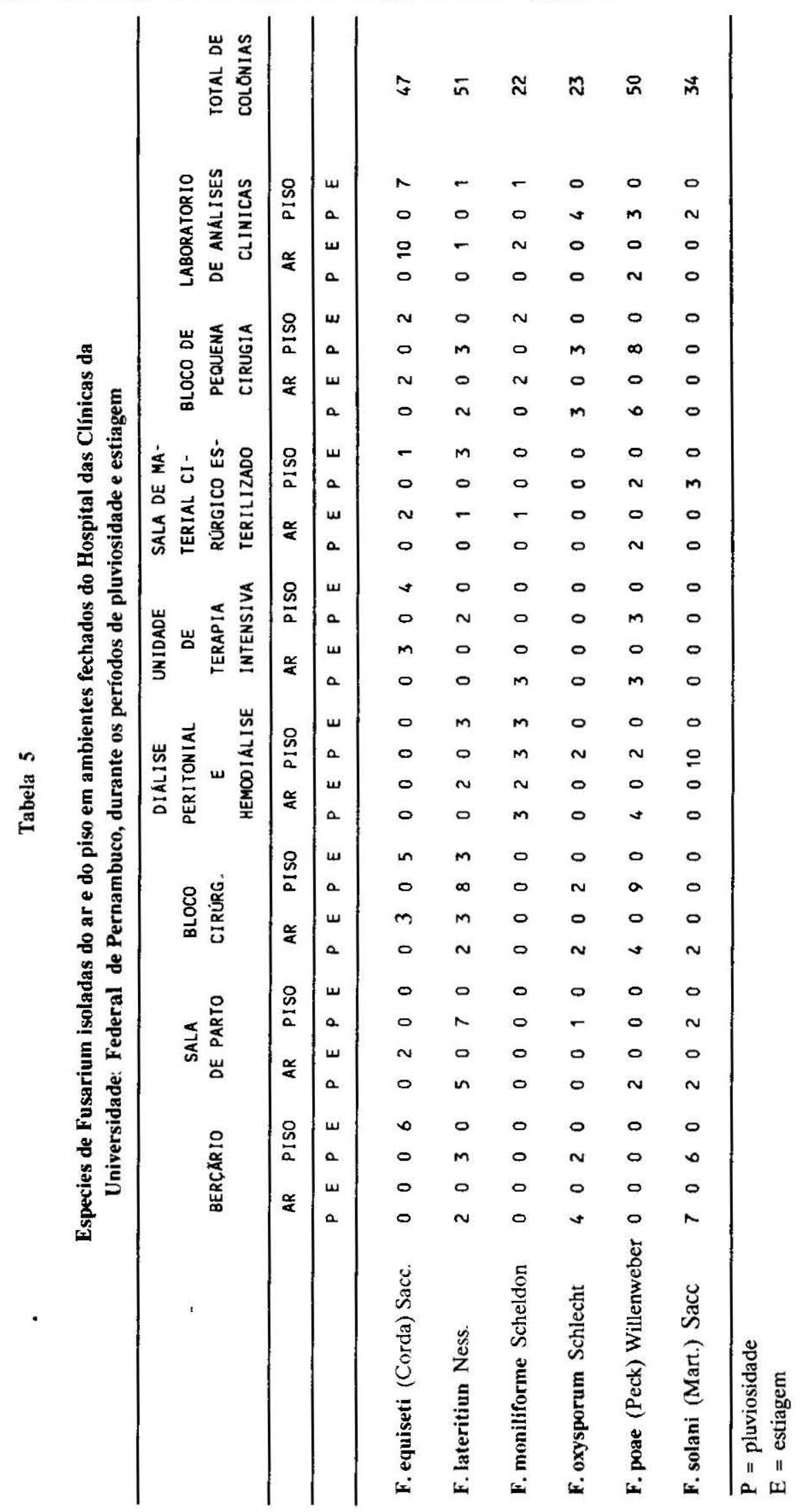




\section{REFERENCIAS}

ABDEL-HAFEZ, S. I. I. \& SHORETT, A. A. M. (1985). Mycotoxins producing fungi and mycoflora af air-dust from Taif. Saudi Arabia. Mycopathologia, Netherlands, 92: 65-71.

ABDEL-HAFEZ, S. I. I.; SHOREIT, A. A. M.; ABDELHAFEZ, A. I. I.; MAGHRABY, O. M. O. E. (1986). Mycoflora and mycotoxin producing fungi of air-dust particles from Egypt. Mycopathologia, Netherlands, 93: 25 32.

ABRAMOWSKY, C. R; QUIN, D; BRADFORD, W. D.; CONANT, N. F. (1974). Sistemic infection by Fusarium in a burned child. The Journal of Pedictries, St. Louis, 84: $561-564$.

ALECRIN, I. \& TEIXEIRA, H. R. (1958). Fungos anemófilos da cidade do Recife (Pernambuco, Brasil). Anais da Faculdade de Medicina do Recife, 18: 269-274.

AUSTWICK, P. K. C. Pathogenicity. In RAPER, K. K. \& FENNEL. L. J. (1965). The genus Aspergillus. Malabar, Fórida Robert E. Trieger. 82-126.

AVILA. R. \& LACEY. J. (1974). The role of Penicillium frequentans in: Suberose (respiratory disease in workers in the rock industri). Clinical Allergy. Oxford, 4: 109-117.

BARKAI-GOLAN. R.: FRANK, M.: KANTON, B.; KARADAVID, R.: TOSHNER, D. (1977). Atmosferic fungi in the desert town of Arad and in the coastal plain of Israel. Annals of Allergy. Minneapolis, 38: 270-274.

BARNETT, J. A.: PAYNE, R. W. \& YARROW. D. (1986). Yeasts: characteristic and identificiation. New York \& Cambrige. 811 p.

BENJAMIN. R. P.: CALlaWAY. J. L.. CONANT, N. F. (1970). Facial granuloma associated with Fusarium infection. Archives of Dermatology, Chicago, 101: 598.

BOOTH. C. (1971). The genus Fusarium. Kew, Surrey, Commonwealth Mycological Institute, 273p.

CALVO, M. A.; CUARRO, J.; SUAREZ, G.; RAMIREZ, C. (1980 a). Airborne fungi in the air of Barcelona (Spain). III. The Genus Aspergilles Link. Mycophatologia, Netherlands. 71: $41-43$.

CARETTA, G.: CRIPPA, A.: DELLA FRANÇA, P.: DELFRATE, G.: GUGLIELMINETT1. M.; MANDIAROTT1, A. M.: PICCO, A. M.; SAVINO. E. (1983). Airborne fungi et Pavia (Italy). Boletin Micológico. Chile. 1: 187-199.
CHU, F. S. (1974). Studies on ochratoxins. Criteria Review Phytopathologie, Wisconsin, 2: 499-524.

CORTEZ, P. J, \& AVILA, R. (1974). Respiratory disease in cork workers (Suberose). Thorax, London, 28 (4); 409-423.

CORTEZ, P. J. \& AVILA, R (1972). Doenças respiratórias dos operários da indústria de cortiça (Suberose). Novos aspectos e possibilidades de diagnóstico. Jomada Médica, Buenos Aires, 84: 16-28.

DAVIS, B. D. (1974). Fungos. In: Microbiologia de Davis. Infecçôes bacterianas e micóticas. $2^{\mathrm{a}}$ ed. Sâo Paulo. Harper \& Row do Brasil Ltda. 3.

DIENER, U. L. \& DAVIS, N. D. (1969). Aflatoxin formation by Aspergillus favus. In: Goldblatt, L. A. Aflatoxin Scientific Back ground. Control and Implications. New York, Academic Press, 13-54.

DORNER, J. W.: COLE, R. J.; DIENER, U. L. (1984). The relationship of Aspergillus navus and Aspergillus parasiticus with reference to production of aflatoxins and cyclopiazomiet acid. Mycopathologia, Netherlands, 87: 1315.

DRANSFIELD, M. (1966). The fungal air spora at Samuru, Northein Nigëria. Transactions British Mycological Society. London, 49: 121-132.

DREXLER, L., KEELAN, M. M.; BONCHER, L. I.: OLINGER, G. N. (1980). Aspergilhus terreus infective endocardites or a procine heterograft valve. Journal Thoracic and Cardiovascular Surgery, Washington, 79: 269274.

ELLIS, M. B. (1971). Dematiaceous. Hyphomycetes. Kew. Surrey Commonwealth Mycological Institue. 608p.

ENGLISH, M. P. (1972), Observations on strains of Fusarium solari and F. oxysporum and Candida parapsilosis from ulcerated degs. Sabouraudia, Edinburg, 10: 35-42.

ESTEVES, J. A.; CABRITA, J. O.; NOBRE, G. N. (1977). Mycologia Médica, Lisboa, Fundaçäo Calouste Gulbenkian.

EVANS, T. \& RUIZ, M: (1955). Mycological flora of the air in San José Costa Rica, Central América. Annals of Allergy. Minneapolis, 13: 189-190

GAMBALE, W.; PURCHIO. A.: GROCE, J. (1977) Flora fúngica anemófila da grande Sâo Paulo. Revista de Microbiologia Sâo Paulo. 8: 74-79. 
Gambale, W.; Purchio, A.; PaUla, C. R (1981) Peridiocidade diária de fungos anemófilos na cidade de Sto Paulo, Brasil. Revista de Microbiologia, Sto Paulo. 12: 176. 181.

GAMBALE, W. \& PURCHIO, A. (1983) Influćncia de fatores abióticos na dispersåo aérea e fungos na cidade de S6o Paulo. Revista de Microbiologia, Sso Paulo, 14: $204-214$.

GEMEINHARDT, H. (1969). Zur Myzologic des LungenAspergilonis. Mykosen, Berlin, 12: 253-281.

GROSE, E. S.: SZEKESSY, M.; MUÑOZ, N. (1967). Airbome fungus spores in Bogotá, Colombia: a five year study. Sabouraudia, Edinburg, 6: 42-50.

HABORN, W. (1960). Aortenruptur durch Asperilltos infection nach operation lines Aorten-stenose. Endoartis polyposa mycotica. Schweizrische medizinische Wochenschrift, Basel, 90: 929-934.

HIRST. J. M. (1953) Chances in atmospheric spore content: diumal periodicity and the effects of the weather. Transactions British Mycological Society. London. 36: 375393.

KRAMER, C. L.; PADY, S. M.; ROGERSON. C. T. (1900). Kansas aeromycology $V$ : Panicitia and Aspertilli. Mycologia, New York, 52: 545-551.

KIDD. G. H. \& WOLF, F. R. (1973). Dimorphism in a pathogenic Fusarium. Mycologia, New York. 65: 1371-1375.

KREGER-VAN RJ, N. J. W. (1984). The Yeasts. A taxomomic study. Amsterdam. Elsevier, 10sop.

LACAZ, C. S.; PORTO, E.: MARTINS. J. E. C. (194). Micologia Médica. $T^{9}$ ed. Sâo Paulo. Sarvier Editora de Livros Médicos Ltda.

LANDECKER, M. E. (1982). Fundamentals of the fungi. New Jersey. Prentice-hall.

LAVERDE, S.; MONCADA, H.; RESTREPO, A.; VERA. C. L. (1973). Micotic keratitis; 5 cases caused by unusus| fungi. Sabouraudia, Edinburg. 11: 119-123.

LIMA, J. A. \& GADELHA. W. (1983). Contaminación de hongos del aire atmosferico en la ciudad de Recife (Pernambuco, Brasil). Revista Latino Americana de Micologia, México, 25: 243-251.

LODDER, J. (1970). The yeasts. A Taxonomic Study. $2^{\mathrm{a}}$ ed. Amsterdam. North-Holland Publishing Company.
MACHADO, G. M. R. Fungos anemófilos do grande Recife. (Tese de Mestrado, 1979).

MARASAS, W. F. O.; THIEL, P. G.; RABIE, C. J. (1986). Moniliformin production in Pusariwan section Liseola. Mycologia, New York, 78 (2) : 242-247.

MONTEMAYOR, L. \& GAMERO, B. H. (1962). Analisis de 6.000 especimes micológicos sintesis estatistica. Comentírios. Mycopathologia et Mycologia Applicata. Den Hag, 18: 1-62.

MORAES, R G.; LETTE. I. C.: GOULART, E. G. (1984). Parasitologia \&icologia Humana. $3^{\mathrm{a}} \mathrm{ed}$. Rio de Janeiro, Editora Cultura Médica.

MOUSTAFA. A. F. \& KAMEL. (1976). A study of fungal populations in the atmosphere of Kuwait. Mycopathologia, Netherlands, 59: 29-35.

NEGRONI, P. \& FISCHER, I. (1942). Fora micológica del aire en Buenos Aires y sus alrededores. Revista del Instituto Bacteriologico. Dr. Carios G. Malbran. Buenos Aires. 11: 228-232.

NEGRONI, P. \& NEGRONI, R. (1984). Micosis Cutâneas y Viscerales, $8^{\mathrm{a}}$ ed. Buenos Aires, Lopes Libreros Editores.

NEGRONI. P.; ROBLES. A. M.; GALUSSIO, J. C. (1972). Estudio comparativo de las reaciones serologicas cuantita. tivas con un antigeno metabólico de Aspergillus fumigatus: Mycopathologia. Netheriands. 48: 275-277.

NELSON, P. E.; TOUSSOUN: T. A. \& MARASAS, W. F. O. (1983). Fusuriom species, an illustrated manual for identification. University Park. Pennsylvania State University Press.

NEWBERNE. P. M. (1974). Mycotoxins: toxicity. carcinogenicity and the influence of various nutritional conditions. Environmental Health Perspectives, Triangle, 9: 1-32.

OLIVEIRA LIMA. A. (1941). Os fungos do ar em alergia respiratória. III. Mêtodos para seu estudo. Brasil Médico, Rio de Janeiro, 55: 693-697.

PADY, S. M. (1957). Quantitative studies of spores in the air. Mycologia, New York. 49: 339-353.

PADY, S. M. KRAMER, C. L.: BONNIE, W. (1962). Kansas aeromyeology XII materials. methods and general results diurnal studies. Mycologia. New York, 54: 168-180.

PANZER. J. D.: TULLIS. E. C.: VAN ARSDEL. E. P. (1957). A simple 24 hours slide spore collector. Phytopathologia, Lancaster. 47: 512-514. 
PASSARELLI, N. (1952). Com que freqüência encontra asmáticos sensiveis a fungos do ar e quais os resultados obtidos com a hipossensibilizaçâo. O Hospital, Rio de Janeiro. 15: 945-947.

PATHAK, V. K. \& PADY, S. S. (1965). Numbers and viability of certain airborne fungus spores. Mycologia, New York, 57: $301-310$

PIRR, J.I.(1985). A Laboratory Guide to Common Penicillium species. CSIRO Division of Food Research, Australia.

PURCHIO, A.; GAMBALE, W.; PAUlA, C. R. (1984). Airborne fungi of Baixada Santista, State of Sâo Paulo-Brazil. Revista de Microbiologia, Sâo Paulo, 15: 258-265.

RAPER, K. B. \& THOM, C. (1949). A manual of Penicillia. Baltimore, Williams and Wilkins. Co.

RAPER, K. B. \& FENNELL, D. I. (1977). The genus Aspergillus. Malabar, Florida. Robert E. Krieger

RIPPON, J. W. (1982). Medical Mycology, $2^{\mathrm{a}}$ ed. Philadelphia Company Saunders W B.

RICHARDSON, K. E.; WINSTON, M.; HAGLER, Jr.; CAMPBELL. C. L.; HAMILTON, P. B. (1985). Production of zearalenone T-2 toxin and deoxynivalenol by Fusarium spp. isolated from plant materials grown in North Carolina. Mycopathologia, Netherlands, 90: 155-160.

ROMERO, H. M. \& RINCON, G. C (1969). Estudio de los hongos atmosféricos de la ciudad de Maracaibo. Kasmera, Venezuela, 3: 89-109.

SAMSON, R. A. (1979). A compilation of the Aspergilli Described Since 1965. Stud. Mycol., Baam, 18: 1-38.

SAFELDER, K. (1980). Atlas of Deep Mycoses. Philadelphia, W. B. Soundameres Company.

SANDHU, D. K. \& SANDHU, R. S. (1973). Survey of Aspergillus species associated with human respiratory tract. Mycopathologia, Netherlands, 49: 77-87.
SEPULVEDA, M. (1985). Hongos y pulmón. Boletin Micológico, Chile, 2: 127-130.

SILVA, M. G.; MOREIRA, Y. K.; CISALPINO, E. O. (1983). Flora fúngica do ar e do piso no Hospital das Clinicas da Universidade Federal de Minas Gerais. Belo HorizonteBrasil. Revista de Microbiologia, Sâo Paulo, 14: 215-222.

STOLK, A. C. \& SAMSON, R. A. (1983). The Ascomycete genus Eupenicillium and related Penicillium anamorphs. Stud. Mycol., Baarn, 23: 1-149.

TANGO, J. C. (1974), Aflatoxina, Boletin do Instituto de Tecnologia de Alimentos, Sâo Paulo, 37: 37-93.

TAYLOR, R. L. \& MCFADDEN, A. W. (1962). Survey of airborne wold flora in Panamá. Mycopathologia, Netherlands, 17: 159-164.

TEIXEIRA. H. R. (1979), Fungos Oportunistas. In: Diagnóstico Laboratorial das Micoses. $2^{\mathrm{a}}$ ed. Recife. Ed da Universidade Federal de Pernambuco.

TORRAS, M. A. C.; ARTIGAS, J. G.; FERNANDES, G. S. (1979). Los hongos como contaminantes en la Industria Farmaceutica Micoflora de los almidones (II) Frecuencia del Gênero Aspergillus: Circular Farmacêutica, Barcelona, 962: 5-8.

VESONDER, R. F., ELLIS, J. J.; ROHWEDDER, W. K. (1981). Elaboration of vomitoxin and zearalenone by Fusarium isolates and the biological activity of Fusarium produced toxins. Applied and Environmental Microbiology, Washington. 42: 1132-1134.

YADAV, A. S. \& MADELIN, M. F. (1968). The ecology of microfungi on decaying stems of Urtiga dioica. Transactions British Mycological Society. 51: 249-259.

ZAIAS, N. (1966). Superficial white onychomycosis. Sabouraudia, Edinburg, 5: 99-103.

ZAPATER, R. C.; ARRECHEA, A.; GUEVARA; V. H. (1972). Queratomicosis por Fusarium dimerum. Sabouraudia, Edinburg, 10: 174-175. 